SLAC-PUB-8084

SU-ITP-99/15

March 20, 1999

\title{
SN1987A Constraints on Large Compact Dimensions
}

\author{
SCHUyler CUlLen円 \\ Physics Department \\ Stanford University, Stanford, California 94309 USA \\ Maxim Perelstein \\ Stanford Linear Accelerator Center \\ Stanford University, Stanford, California 94309 USA
}

\begin{abstract}
Recently there has been a lot of interest in models in which gravity becomes strong at the $\mathrm{TeV}$ scale. The observed weakness of gravitational interactions is then explained by the existence of extra compact dimensions of space, which are accessible to gravity but not to Standard Model particles. In this letter we consider graviton emission into these extra dimensions from a hot supernova core. The phenomenology of SN1987A places strong constraints on this energy loss mechanism, allowing us to derive a bound on the fundamental Planck scale. For the case of two extra dimensions we obtain a very strong bound of $\mathrm{M} \gtrsim 50$ $\mathrm{TeV}$, which corresponds to a radius $\mathrm{R} \lesssim 0.3 \mu \mathrm{m}$. While there are a lot of sources of uncertainty associated with this bound, we find that pushing it down to the few- $\mathrm{TeV}$ range, which could in principle be probed by ground-based experiments, is disfavored. For three or more extra dimensions the SN1987A constraints do not exclude a $\mathrm{TeV}$ gravitational scale.
\end{abstract}

\footnotetext{
${ }^{1}$ Work partially supported by NSF grant PHY-9870115.

${ }^{2}$ Work supported by the Department of Energy, contract DE-AC03-76SF00515.
} 
Recently, Arkani-Hamed, Dimopoulos and Dvali proposed a novel solution to the hierarchy problem, which does not rely on either low-energy supersymmetry or compositeness of the Higgs boson [1, 2, 3]. They pointed out that if there exist new compact spatial dimensions, the fundamental (higher-dimensional) Planck scale $M$ could be close to the electroweak scale $M_{\mathrm{EW}}$, thus avoiding the hierarchy. That is, gravity could become comparable in strength to other interactions at energies of $\mathrm{TeV}$ order. At distances large compared to the size of the compact dimensions, gravity obeys the 4-dimensional Newton's Law, with the gravitational constant given by

$$
G_{N}=\frac{1}{4 \pi} M^{-n-2} R^{-n}
$$

where $n$ is the number of extra dimensions, and $R$ is their size In order to reproduce the measured value $G_{N}=6.7 \times 10^{-39} \mathrm{GeV}^{-2}$ with the fundamental scale $M \sim 1 \mathrm{TeV}$, we require

$$
R \sim 2 \times 10^{31 / n-17} \mathrm{~cm}
$$

The most obvious experimental consequence of this scenario is the violation of Newton's Law at distances of order $R$. Macroscopic measurments of gravity constrain $R$ to be less than about a millimeter [4]. From (11) we see that for $n=1, R \sim 10^{13} \mathrm{~cm}$, so this case is clearly excluded. For $n \geq 2$, however, this constraint is satisfied: $M=1 \mathrm{TeV}$ corresponds to $R=0.68 \mathrm{~mm}$ for $n=2$ and to $R=3.0 \times 10^{-12} \mathrm{~cm}$ for $n=6$.

Since the Standard Model provides an accurate description of the strong, electromagnetic and weak processes up to energies of order $100 \mathrm{GeV}$, the quarks, leptons and gauge bosons cannot propogate in the extra dimensions. Therefore they must be localized to a 4-dimensional hypersurface within the full space-time. While such a localization may be achieved by purely field-theoretic mechanisms [1], the most attractive possibility is provided by D-branes of string theory [2], since they naturally appear with certain degrees of freedom confined to them.

If indeed gravity becomes strong at $\mathrm{TeV}$ scale, the higher-dimensional gravitons should have significant couplings to the Standard Model particles at energies accessible to current and near-future collider experiments [3]. In particular, graviton production in $e^{+} e^{-}$and $p \bar{p}$ collisions leads to events with missing energy [5, 6]. The current results from searches for such events at LEP and Tevatron require that $M$ be higher than $1.2 \mathrm{TeV}$ for $n=2$ and $610 \mathrm{GeV}$ for $n=6$ [6]. A number of authors have also considered the effects of the virtual graviton exchange on various observables [5, 7], as well as graviton loop effects on the anomalous magnetic moment of the muon [8].

Various astrophysical and cosmological processes can also be used to put constraints on the model. In particular, the order-of-magnitude estimates in [3] show that the agreement of the observed neutrino fluxes from the supernova SN1987A with the predictions of the stellar collapse models [11] requires that the fundamental scale be as high as $M \sim 30 \mathrm{TeV}$ for $n=2$. Since this is one of the most stringent bounds known on $M$, we feel that a more detailed

* Throughout this paper, we will assume for simplicity that the new dimensions are compactified on a torus of periodicity $2 \pi R$ in each direction. 
quantitative study of graviton emission from the supernova is warranted. In particular, the next-generation collider experiments, as well as (for the case of two extra dimensions) the upcoming precision measurements of gravity on short distances, could probe values of $M$ up to a few TeV [4, 5, 6]. Therefore it is interesting to ask whether this range is already ruled out by SN1987A. We will address this question in this letter.

According to the standard theory of type-II supernovae, most of the $\sim 10^{53}$ ergs of gravitational binding energy released during core collapse is carried away by neutrinos. This hypothesis was essentially confirmed by the measurements of neutrino fluxes from SN1987A by Kamiokande [9] and IMB [10] collaborations. These measurements allow one to put powerful constraints on new physics. Indeed, if there is some novel channel through which the core of the supernova can lose energy, the luminosity in this channel should be low enough to preserve the agreement of neutrino observations with theory. This idea was used to put the strongest experimental upper bounds on the axion mass [12]. Here, we will consider the emission of the higher-dimensional gravitonsil] from the core. Once these particles are produced, they escape into the extra dimensions, carrying energy away with them. The constraint on the luminosity of this process can be converted into a bound on the fundamental Planck scale of the theory, $M$.

During the first few seconds after collapse, the core contains neutrons, protons, electrons, neutrinos and thermal photons. There are a number of processes in which higher-dimensional gravitons can be produced. For the conditions that pertain in the core at this time (temperatures $T \sim 30-70 \mathrm{MeV}$, densities $\left.\rho \sim(3-10) \times 10^{14} \mathrm{~g} \mathrm{~cm}^{-3}\right)$, the dominant process is nucleon-nucleon "gravi-strahlung",

$$
N+N \rightarrow N+N+G
$$

where $N$ can be a neutron $n$ or a proton $p$, and $G$ is a higher-dimensional graviton. Electromagnetic processes in which gravitons can be emitted, such as ep $\rightarrow e p G$ and $e e \rightarrow e e G$, are much less important at temperatures of interest than the strong force mediated reaction (2). The processes involving photons, $\gamma \gamma \rightarrow G, \gamma e \rightarrow e G$ and $\gamma p \rightarrow p G$, are also suppressed, since the photons have significantly smaller number density than nucleons. In this paper, we will only consider reaction (2).

To model the nucleon-nucleon interaction, we have employed the one-pion exchange approximation. The nucleon-pion interaction Lagrangian is

$$
\mathcal{L}_{N N \pi}=-\frac{i g_{A}}{F_{\pi}} \partial_{\mu} \pi^{a} \bar{N} \gamma_{5} \gamma^{\mu} \tau^{a} N
$$

where $N^{T}=(p, n), \tau^{a}$ are Pauli matrices, $g_{A} \simeq 1.25$, and $F_{\pi} \simeq 180 \mathrm{MeV}$. While the validity of the one-pion exchange potential at nuclear densities is questionable, we believe it

\footnotetext{
${ }^{\dagger}$ It is likely that, in realistic models, the extra dimensions will contain scalar, vector, and even fermion fields that have substantial couplings to matter [13]. While these particles could also contribute to the energy loss from the core and strenghten our bound, we do not consider them here, since this contribution is model-dependent.
} 


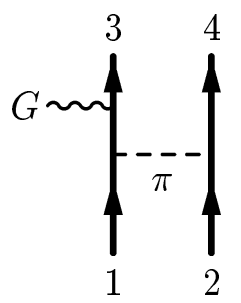

(a)

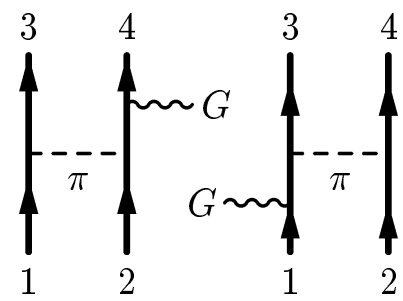

(b) (c)

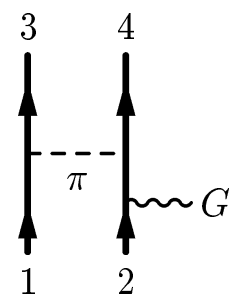

(d)

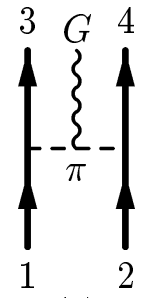

(e)

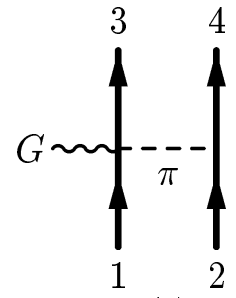

(f)

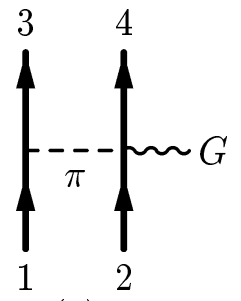

(g)

Figure 1: Diagrams that contribute to the gravi-strahlung process in nucleon-nucleon collision in the one-pion exchange approximation.

is appropriate to use it for our present purposes, since factors of order unity in the graviton emission rate will not have much effect on our conclusions.

From the four-dimensional point of view, a higher-dimensional graviton appears as a tower of "Kaluza-Klein modes", massive particles whose mass is determined by their momentum in the extra dimensions and is quantized in units of $1 / R$. The typical energies at which gravitons are emitted in the core are much lower than the fundamental scale; in this limit, only spin-2 modes (which we will refer to as "gravitons", $h^{\mu \nu}$ ) and spin-0 modes ("dilatons", $\phi)$ couple to the Standard Model particlesf. The Lagrangian is [15]:

$$
\mathcal{L}=-\frac{\kappa}{2} \sum_{\vec{j}}\left(h^{\mu \nu, \vec{j}} T_{\mu \nu}+\omega \phi^{\vec{j}} T_{\mu}^{\mu}\right),
$$

where $T_{\mu \nu}$ is the conserved energy-momentum tensor of the matter, $\kappa=\sqrt{32 \pi G_{N}}, \omega=$ $\sqrt{\frac{2}{3(n+2)}}$, and the sum is over the various Kaluza-Klein $(\mathrm{KK})$ modes, labelled by their momentum in the compact dimensions $\vec{j}$. Note that each KK mode couples to matter with the usual four-dimensional gravitational strength. However, for $n \leq 4$, the number of these modes that can be emitted at the core temperatures is very large: the mass splitting between the modes ranges from $10^{-4} \mathrm{eV}$ for $n=2$ to about $10 \mathrm{keV}$ for $n=4$. This enormous multiplicity leads to enhancement of their effects, and allows us to put strong constraints on the model. For $n>4$, the mass splitting becomes comparable to the temperatures in the core, so that only the first few modes can be emitted. In this case, the bound we obtain is very weak, and we will not discuss it here. Note also that once a particular mode is emitted,

\footnotetext{
†This fact is obvious from the effective field theory point of view, developed in 14 .
} 
it practically does not interact with matter. Thus, the gravitons, unlike the axions and neutrinos, cannot be trapped in the core. We emphasize that the Lagrangian (4) is completely independent of the details of the physics at the fundamental scale $M$. Therefore, our results will provide a model-independent bound on this scale.

Given the Lagrangians (3) and (㺼), it is a simple matter to evaluate the matrix elements for each of the processes in (2). There are 14 diagrams to evaluate, the seven "direct" diagrams shown in Figure 1, and seven "exchange" diagrams, with legs 3 and 4 interchanged. Since the temperatures in the core are of order $30-70 \mathrm{MeV}$, the nucleons are nonrelativistic. Naively, one would expect the diagrams (a)-(d) (and the corresponding exchange diagrams) to dominate in this regime. However, the leading contributions cancel due to energy and momentum conservation, and all the diagrams have to be included in the calculation. We have also made the approximation that $m_{\pi}^{2} \ll 3 m_{N} T$; this is satisfied for $T \gg 6 \mathrm{MeV}$. For the emission of a single Kaluza-Klein graviton mode $\vec{j}$ we find

$$
\begin{gathered}
\sum_{\text {spin }}\left|\mathcal{M}^{\vec{j}}(n n \rightarrow n n G)\right|^{2}=\sum_{\text {spin }}\left|\mathcal{M}^{\vec{j}}(p p \rightarrow p p G)\right|^{2}=\mathcal{A}^{\vec{j}}+\mathcal{B}^{\vec{j}}-2 \mathcal{C}^{\vec{j}}, \\
\sum_{\text {spin }}\left|\mathcal{M}^{\vec{j}}(n p \rightarrow n p G)\right|^{2}=\mathcal{A}^{\vec{j}}+4 \mathcal{B}^{\vec{j}}+4 \mathcal{C}^{\vec{j}}
\end{gathered}
$$

where $\mathcal{A}^{\vec{j}}$ and $\mathcal{B}^{\vec{j}}$ are the contributions of the direct and exchange diagrams, respectively, and $\mathcal{C}^{j}$ is due to the interference between the two types of diagrams. A minus sign appears in the first line due to exchange of identical fermions in the final state. In $n p$ scattering, the contributions of exchange diagrams are enhanced because they involve charged pions, which couple to nucleons more strongly than neutral pions by a factor of $\sqrt{2}$. Explicitly, we find

$$
\begin{aligned}
& \mathcal{A}^{\vec{j}}= \mathcal{B}^{\vec{j}} \\
&=\frac{1024 \pi}{45} \frac{m_{N}^{4} g_{A}^{4} G_{N}}{F_{\pi}^{4}}\left[27+26 \frac{m_{N}^{2} m_{g}^{2}}{(\vec{k} \cdot \vec{l})^{2}}+7 \frac{m_{N}^{4} m_{g}^{4}}{(\vec{k} \cdot \vec{l})^{4}}+\frac{|\vec{k}|^{2}|\vec{l}|^{2}}{(\vec{k} \cdot \vec{l})^{2}}\left(19+22 \frac{m_{N}^{2} m_{g}^{2}}{(\vec{k} \cdot \vec{l})^{2}}+4 \frac{m_{N}^{4} m_{g}^{4}}{(\vec{k} \cdot \vec{l})^{4}}\right)\right], \\
& \mathcal{C}^{\vec{j}=-} \frac{512 \pi}{45} \frac{m_{N}^{4} g_{A}^{4} G_{N}}{F_{\pi}^{4}}\left[27+26 \frac{m_{N}^{2} m_{g}^{2}}{(\vec{k} \cdot \vec{l})^{2}}+7 \frac{m_{N}^{4} m_{g}^{4}}{(\vec{k} \cdot \vec{l})^{4}}-5 \frac{(\vec{k} \cdot \vec{l})^{2}}{|\vec{k}|^{2}|\vec{l}|^{2}}\left(7+6 \frac{m_{N}^{2} m_{g}^{2}}{(\vec{k} \cdot \vec{l})^{2}}+2 \frac{m_{N}^{4} m_{g}^{4}}{(\vec{k} \cdot \vec{l})^{4}}\right)\right. \\
&\left.+\left(\frac{|\vec{k}|^{2}|\vec{l}|^{2}}{(\vec{k} \cdot \vec{l})^{2}}-4 \frac{(\vec{k} \cdot \vec{l})^{4}}{|\vec{k}|^{4}|\vec{l}|^{4}}\right)\left(19+22 \frac{m_{N}^{2} m_{g}^{2}}{(\vec{k} \cdot \vec{l})^{2}}+4 \frac{m_{N}^{4} m_{g}^{4}}{(\vec{k} \cdot \vec{l})^{4}}\right)\right] ;
\end{aligned}
$$

where $\vec{k}=\overrightarrow{p_{1}}-\overrightarrow{p_{3}}, \vec{l}=\overrightarrow{p_{1}}-\overrightarrow{p_{4}}$, and $m_{g}$ is the four-dimensional mass of the emitted graviton mode. These expressions have been averaged over the direction of the graviton. We have also evaluated the matrix elements for the dilaton emission, but since their contribution to the energy loss rate turns out to be negligible we do not present them here.

The energy loss rate for the nucleon-nucleon gravi-strahlung is given by the following phase-space integral:

$$
\dot{\epsilon}=\sum_{\vec{j}} \int d \Pi_{1} d \Pi_{2} d \Pi_{3} d \Pi_{4} d \Pi_{g} S\left|\mathcal{M}^{\vec{j}}\right|^{2}(2 \pi)^{4} \delta^{4}\left(p_{1}+p_{2}-p_{3}-p_{4}-p_{g}\right) E_{g} f_{1} f_{2}\left(1-f_{3}\right)\left(1-f_{4}\right),
$$


where $d \Pi_{i}=d^{3} p_{i} /(2 \pi)^{3} 2 E_{i}$, the labels $i$ denote the incoming $(i=1,2)$ and outgoing $(i=3,4)$ nucleons, the label $g$ denotes the higher-dimensional graviton, $S$ is the symmetry factor for identical particles in the initial and final states $(S=1 / 4$ for $n n \rightarrow n n, p p \rightarrow p p$, and $S=1$ for $n p \rightarrow n p)$, and $f_{i}=\left[\exp \left(E_{i} / T-\mu_{i} / T\right)+1\right]^{-1}$ are the distribution functions of the nucleons. Since the typical energies in our process are much higher than the splitting of the KK modes, we can replace the sum over these modes by an integral over the four-dimensional mass, according to

$$
\sum_{\vec{j}} \rightarrow R^{n} \int d^{n} m=\frac{1}{2} \Omega_{n} R^{n} \int\left(m^{2}\right)^{(n-2) / 2} d m^{2}=\frac{\Omega_{n}}{8 \pi} M^{-(n+2)} G_{N}^{-1} \int\left(m^{2}\right)^{(n-2) / 2} d m^{2},
$$

where $\Omega_{n}$ is the surface area of the unit sphere in $n$ dimensions.

We will assume that the nucleons in the core are non-degenerate; in this limit, $f_{i}=$ $\exp \left(\mu_{i} / T-E_{i} / T\right)$ and we can neglect the "blocking factors", $1-f_{3}$ and $1-f_{4}$. Integrating the matrix elements (5) over the phase-space in (7) and over the four-dimensional mass of the emitted graviton, we get

$$
\begin{aligned}
& \dot{\epsilon}=1.7 \times 10^{17} \mathrm{erg} \mathrm{g}^{-1} \mathrm{~s}^{-1}\left(X_{n}^{2}+X_{p}^{2}+7.0 X_{n} X_{p}\right) \rho_{14} T_{\mathrm{MeV}}^{5.5} M_{\mathrm{TeV}}^{-4}, \quad n=2 ; \\
& \dot{\epsilon}=9.4 \times 10^{11} \mathrm{erg} \mathrm{g}^{-1} \mathrm{~s}^{-1}\left(X_{n}^{2}+X_{p}^{2}+7.8 X_{n} X_{p}\right) \rho_{14} T_{\mathrm{MeV}}^{6.5} M_{\mathrm{TeV}}^{-5}, \quad n=3 ; \\
& \dot{\epsilon}=5.9 \times 10^{6} \operatorname{erg~g}^{-1} \mathrm{~s}^{-1}\left(X_{n}^{2}+X_{p}^{2}+8.8 X_{n} X_{p}\right) \rho_{14} T_{\mathrm{MeV}}^{7.5} M_{\mathrm{TeV}}^{-6}, \quad n=4 ;
\end{aligned}
$$

where $X_{p}$ and $X_{n}=1-X_{p}$ are the proton and neutron fractions in the core, $\rho_{14}=$ $\rho /\left(10^{14} \mathrm{~g} \mathrm{~cm}^{-3}\right), T_{\mathrm{MeV}}=T /(1 \mathrm{MeV})$, and $M_{\mathrm{TeV}}=M /(1 \mathrm{TeV})$.

In order to obtain reliable bounds on the fundamental Planck scale $M$, one would have to incorporate the luminosities (9) into a numerical code for a protoneutron star evolution, and calculate the expected neutrino fluxes for various values of $M$. For the purposes of this paper, however, we will adopt a simple analytic criterion, suggested by Raffelt (see p. 504 of [11].) Namely, we will require that the energy loss rate to gravitons, evaluated at typical core conditions, does not exceed $10^{19} \mathrm{erg} \mathrm{g}^{-1} \mathrm{~s}^{-1}$. Using (9) then yields, for the case of two extra dimensions,

$$
M>0.36 \mathrm{TeV}\left(X_{n}^{2}+X_{p}^{2}+7.0 X_{n} X_{p}\right)^{0.25} \rho_{14}^{0.25} T_{\mathrm{MeV}}^{1.375}, \quad n=2 .
$$

This bound depends rather weakly on the proton fraction, core density, and the exact value of the maximum luminosity we allow. On the other hand, its temperature dependence is strong. Therefore, the main uncertainty comes from the lack of precise knowledge of temperatures in the core: values quoted in the literature range from 30 to $70 \mathrm{MeV}$. For a numerical estimate, we have assumed, conservatively, $T=30 \mathrm{MeV}, \rho=3 \times 10^{14} \mathrm{~g} \mathrm{~cm}^{-3}$, and $X_{p}=0$. This choice of parameters yields

$$
M \gtrsim 50 \mathrm{TeV}, \quad n=2 .
$$

This corresponds to an extra dimension size of

$$
R \lesssim 3 \times 10^{-4} \mathrm{~mm}, \quad n=2 .
$$


These values of $M$ and $R$ are well beyond the reach of both current and near-future groundbased experiments. We note that for higher realistic values of temperatures and densities in the core the bound obtained from (10) can be as high as $250 \mathrm{TeV}$.

In the course of our calculation, we have made a number of simplifying approximations. In particular, we have not considered multiple-scattering effects, which suppress emission of gravitons with energies below the nucleon-nucleon collision rate in the medium $\$$ The deviations of the nucleon mass and nucleon-pion coupling from their vaccuum values in the dense medium of the core were also neglected. While at the core temperatures the nucleons are neither strongly degenerate nor non-degenerate, we have not included the degeneracy effects, such as the Pauli blocking factors for the final-state nucleons. One may also worry about the inadequacies of the one-pion exchange approximation at short distances, e.g. the effects of pion loop diagrams, other meson exchanges, and the hard core of the nucleonnucleon potential. Finally, we have not considered the possibility that novel phases of nuclear matter (pion condensates, quark-gluon plasma, superfluidity, etc.) may form in certain regions of the core.

To make a crude estimate of the uncertainties introduced by neglecting the above effects, we used the results for the very similar process of nucleon-nucleon axion bremstrahlung, which has been studied in great detail. For this process, it was shown that a simple calculation based on the same approximations we have made here gives a good first estimate of the bound on axion-nucleon coupling, $g_{a} \lesssim 10^{-10}$ [11]. This is only about a factor of 3 less restrictive than the generally accepted bound, corresponding to about a factor of 10 uncertainty in the axion luminosity. We expect a similar result to hold for the process considered here, even though a much more careful study is of course necessary to confirm it. If this is the case, our bound on $M$ is quite robust, since changing the graviton luminosity by a factor of 10 only changes the bound by $10^{0.25} \simeq 1.8$. Note that in order to push this bound down to the range of a few $\mathrm{TeV}$, which in principle could be probed by both collider and gravitational experiments, our naive calculation would have to overestimate the luminosity by more than 3 orders of magnitude. We feel therefore that it is fair to say that this range is disfavored, and likely ruled out, by SN1987A.

We have repeated our analysis for $n=3$ and $n=4$. With the same parameter choices as before, we obtain the bounds:

$$
\begin{array}{lll}
M \gtrsim 4 \mathrm{TeV}, & R \lesssim 4 \times 10^{-7} \mathrm{~mm}, & n=3 ; \\
M \gtrsim 1 \mathrm{TeV}, & R \lesssim 2 \times 10^{-8} \mathrm{~mm}, & n=4 .
\end{array}
$$

While these bounds are quite strong, our conclusions in this case are much more optimistic. Given the uncertainties of our analysis, models with large extra dimensions and values of the fundamental scale which could be probed by near-future collider experiments are certainly not excluded for $n \geq 3$.

${ }^{\S}$ This phenomenon is analogous to the Landau-Pomeranchuk-Migdal effect 16 in the usual photon bremstrahlung. 
In summary, we have found that graviton emission from SN1987A puts very strong constraints on models with large extra dimensions in the case $n=2$. In this case, for a conservative choice of the core parameters we arrive at a bound on the fundamental Planck scale $M \gtrsim 50 \mathrm{TeV}$, which corresponds to a radius $\mathrm{R} \lesssim 0.3 \mu \mathrm{m}$. Even though taking into account various uncertainties encountered in our calculation can weaken this bound, it is unlikely that it can be pushed down to the phenomenologically interesting range of a few TeV. For $n=3$ and 4, we find that the fundamental scale has to be higher than about 4 and $1 \mathrm{TeV}$, respectively.

We are grateful to Nima Arkani-Hamed, Savas Dimopoulos, Lance Dixon, Michael Peskin, Georg Raffelt, Scott Thomas and Robert Wagoner for useful discussions. M. P. was supported by the Department of Energy under contract DE-AC03-76SF00515. S. C. was partially supported by NSF grant PHY-9870115. 


\section{References}

[1] N. Arkani-Hamed, S. Dimopoulos, and G. Dvali, Phys. Lett. B 429, 263 (1998).

[2] I. Antoniadis, N. Arkani-Hamed, S. Dimopoulos, and G. Dvali, Phys. Lett. B 436, 257 (1998).

[3] N. Arkani-Hamed, S. Dimopoulos, and G. Dvali, Phys. Rev. D 59, 086004 (1999).

[4] Macroscopic bounds on modifications of gravity have recently been reviewed by J. C. Long, H. W. Chan, and J. C. Price, Nucl. Phys. B 539, 23 (1999).

[5] G. F. Giudice, R. Rattazzi, and J. D. Wells, Nucl. Phys. B 544, 3 (1999).

[6] E. A. Mirabelli, M. Perelstein, and M. E. Peskin, Phys. Rev. Lett. 82, 2236 (1999).

[7] J. L. Hewett, hep-ph/9811356; P. Mathews, S. Raychaudhuri, and K. Sridhar, hepph/9812486; T. G. Rizzo, hep-ph/9901209; K. Agashe, N. G. Deshpande, hep$\mathrm{ph} / 9902263$.

[8] M. L. Graesser, hep-ph/9902310.

[9] K. Hirata et al., Phys. Rev. Lett. 58, 1490 (1987).

[10] R. M. Bionta et al., Phys. Rev. Lett. 58, 1494 (1987).

[11] G. G. Raffelt, Stars as Laboratories for Fundamental Physics (Chicago, USA: Univ. Pr., 1996).

[12] Originally, the SN1987A bounds on the axion mass were discussed in R. Mayle et al., Phys. Lett. B 203, 188 (1988); G. G. Raffelt and D. Seckel, Phys. Rev. Lett. 60, 1793 (1988); M. S. Turner, Phys. Rev. Lett. 60, 1797 (1988). Subsequently, a lot of work has been done on refining these bounds. For reviews, see G. G. Raffelt, Phys. Rep. 198, 1 (1990), and Ref. [11].

[13] N. Arkani-Hamed, S. Dimopoulos, hep-ph/9811353.

[14] R. Sundrum, Phys. Rev. D 59, 085009 (1999).

[15] T. Han, J. D. Lykken, and R.-J. Zhang, hep-ph/9811350.

[16] L. D. Landau and I. Pomeranchuk, Dokl. Akad. Nauk. SSSR 92, 535 (1953), and 92, 735 (1953); A. B. Migdal, Phys. Rev. 103, 1811 (1956). 